\title{
A Case of Transplant Nephrectomy due to Chronic Graft Intolerance Syndrome
}

\author{
Masahiro Tomonari ${ }^{a}$ Akimitsu Kobayashia $^{a}$ Izumi Yamamoto $^{\mathrm{a}}$ \\ Saeko Hatanaka ${ }^{a}$ Mayuko Kawabe ${ }^{a}$ Takafumi Yamakawa ${ }^{a}$ Haruki Katsumata ${ }^{a}$ \\ Ai Katsuma $^{a}$ Aki Mafune ${ }^{a}$ Yasuyuki Nakada $^{a}$ Yusuke Koike $^{\mathrm{b}}$ Jun Miki ${ }^{\mathrm{b}}$ \\ Takahiro Kimura $^{b}$ Yudo Tanno $^{\text {a }}$ Hiroyasu Yamamoto $^{\text {a }}$ Takashi Yokoo $^{\text {a }}$ \\ aDivision of Nephrology and Hypertension, Department of Internal Medicine, The Jikei University School of \\ Medicine, Tokyo, Japan; bepartment of Urology, The Jikei University School of Medicine, Tokyo, Japan
}

\section{Keywords}

Transplant nephrectomy · Graft intolerance syndrome ·

Allograft rejection

\begin{abstract}
We report a case of graft intolerance syndrome in which transplant nephrectomy was performed 11 years after kidney transplantation. A 46-year-old man was admitted to our hospital in February 2018 with a mild fever, left lower abdominal pain, and gross hematuria with enlargement of the transplanted kidney. Urinary tract infection was ruled out. Because the symptoms developed after the immunosuppressants had been stopped after kidney graft loss, graft intolerance syndrome was suspected. He had lost his graft in 2016 and had stopped all immunosuppressants since January of 2017. Immunosuppressive therapy was intensified, and steroid half-pulse therapy was added for 3 days. After the steroid pulse therapy, the C-reactive protein (CRP) decreased from $6.47 \mathrm{mg} / \mathrm{dL}$ to $0.76 \mathrm{mg} / \mathrm{dL}$, but there was little improvement in the symptoms, and the CRP then increased to $4.44 \mathrm{mg} / \mathrm{dL}$. Transplant nephrectomy was performed in March 2018. Postoperatively, the symptoms disappeared without the administration of immunosuppressants, and the
\end{abstract}

CRP decreased. Pathologically, the resected kidney graft showed persistent active allograft rejection with severe endarteritis, transplant glomerulopathy, and diffuse interstitial fibrosis. Massive thrombi occluded the large arteries, and there was extensive hemorrhagic cortical necrosis. Transplant nephrectomy is uncommon in patients $>6$ months after transplantation. However, even if more time has passed since transplantation, as in this case, transplant nephrectomy may be a valid option in some cases of severe graft intolerance syndrome.

(c) 2020 S. Karger AG, Basel

\section{Background}

About 10\% of all kidney allografts fail in the first year of transplantation, after which the rate is $3-5 \%$ annually $[1,2]$. Transplanted renal failure induces an inflammatory response and is associated with increased morbidity and mortality [3-5].

Transplant nephrectomy has advantages and disadvantages. Transplant nephrectomy after graft loss can improve the prognosis [6], while the preserved kidney has residual function, which helps reduce erythropoiesis-

$\begin{aligned} & \text { karger@karger.com } \\ & \text { www.karger.com/nef }\end{aligned}$
Karger ${ }^{\prime /}$




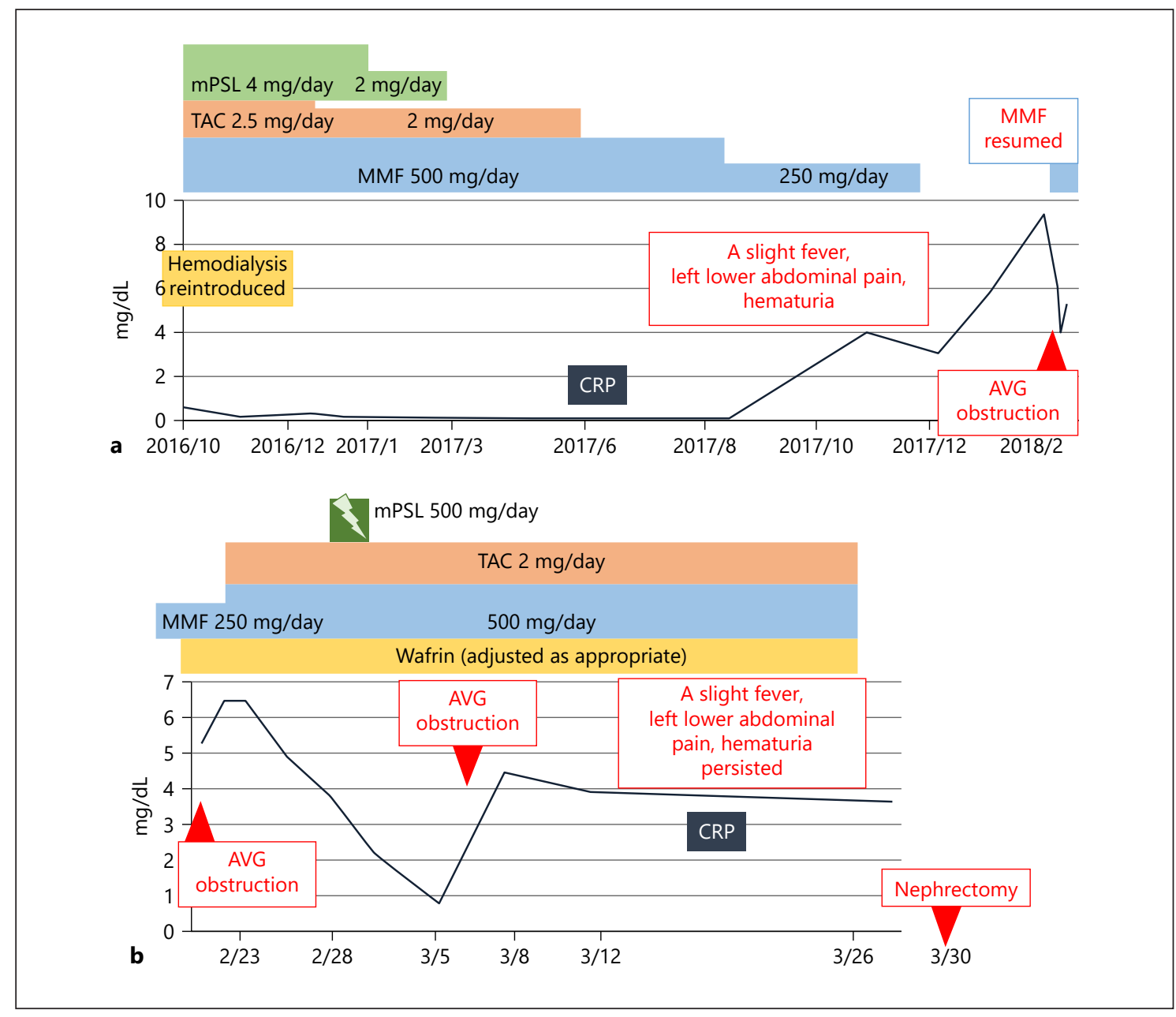

Fig. 1. Clinical course. mPSL, TAC, MMF, CRP, and AVG. a Prehospital clinical course. b Clinical course after admission. mPSL, methylprednisolone; TAC, tacrolimus hydrate; MMF, mycophenolate mofetil; CRP, C-reactive protein; AVG, arteriovenous graft.

stimulating agent and fluid intake restriction [7]. In addition, transplant nephrectomy is detrimental to the prognosis for subsequent transplantation because of increased antibody levels to incompatible antigens and is associated with greater risks of death and morbidity after late graft failure [9]. Consequently, transplant nephrectomy is rarely performed in patients who have lost kidney graft function $>6$ months after transplantation [10]. Although there are no large clinical trials and the indications for transplant nephrectomy remain controversial, one of the major reasons for transplant nephrectomy after late graft failure is graft intolerance syndrome, defined as a condition in which the patient is unable to tolerate allografted kidneys after their loss of function. Here, we report a case of graft intolerance syndrome in which transplant ne-

Transplant Nephrectomy due to Chronic Graft Intolerance Syndrome phrectomy was performed 11 years after kidney transplantation, and the symptoms improved with no postoperative complications. This case should lead to debate about the indications for transplant nephrectomy in patients with graft intolerance syndrome, and we also discuss the pathological findings of the resected kidney graft in detail.

\section{Case Report}

A 46-year-old man was admitted to our hospital in February 2018 because of a mild fever, left lower abdominal pain, and gross hematuria. He had undergone peritoneal dialysis due to end-stage kidney disease caused by IgA nephropathy when he was 34 years old. The next year, he received an ABO-compatible living-related 

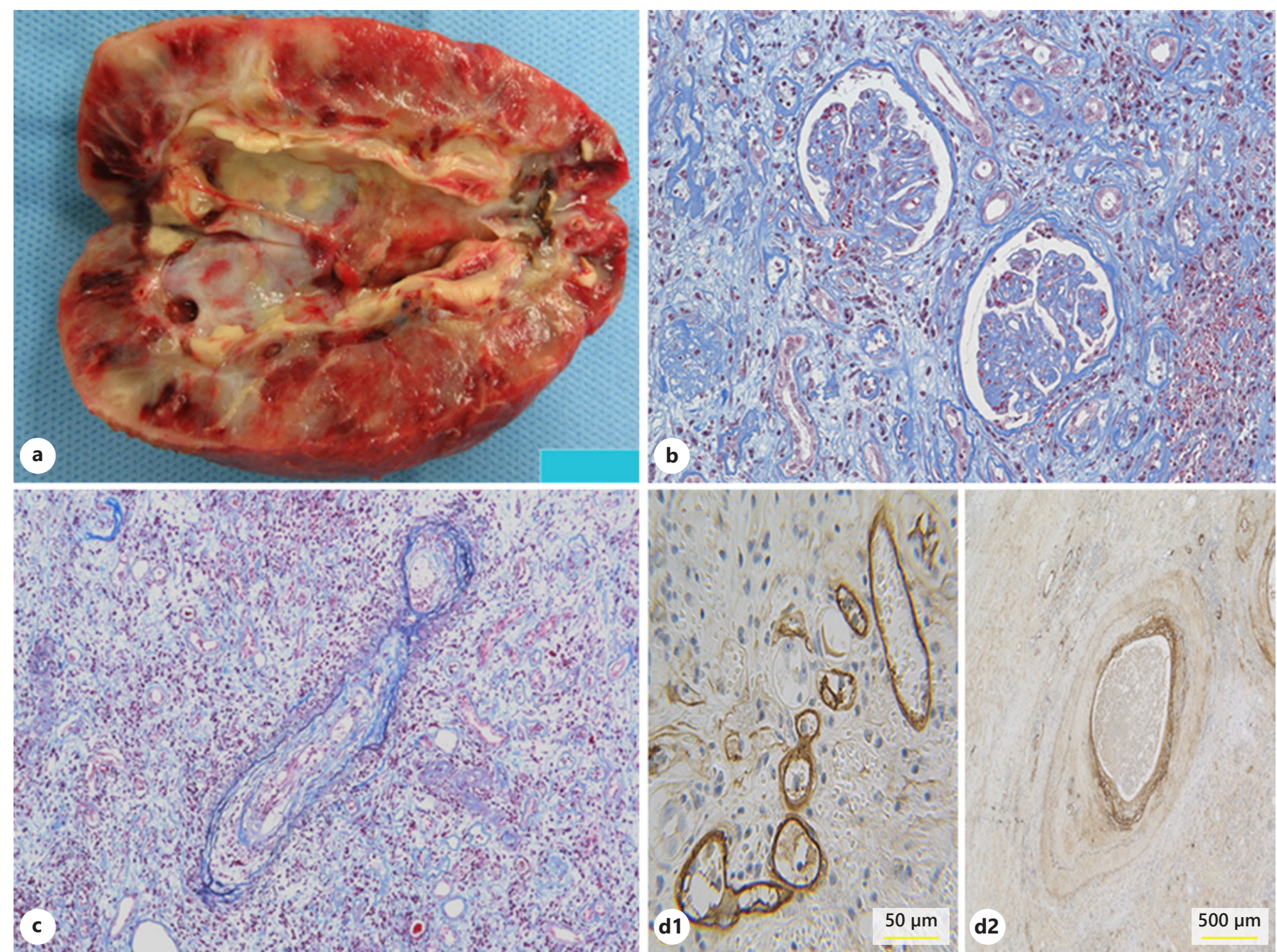

Fig. 2. a The gross pathology showed white areas of degeneration and hemorrhage suggesting necrosis in the parenchyma; the parenchyma was weakened in places. b Severe transplant glomerulopathy was identified in the residual glomeruli (Masson, $\times 200$ ).

kidney transplant from his father. Three years later, the recurrence of IgA nephropathy and chronic antibody-mediated rejection were confirmed by a kidney graft biopsy. Renal biopsy results are as follows. Two of 15 glomeruli showed global sclerosis, and 3 cellular crescents and 1 fibrous crescent were observed. A small area of fibrinoid necrosis was identified in the glomerular capillary. An immunohistological study showed mesangial and peripheral IgA deposits. Electron microscopy showed electron-dense deposits in the mesangial and paramesangial area, and mild peritubular capillary basement membrane multilayering and thickening. During the hospitalization, we also checked on donor-specific antibodies using Luminex technology and detected HLA Class I, B67. Steroid pulse therapy and a tonsillectomy were performed, and the immunosuppressive agents were strengthened. Nevertheless, there was no improvement in renal function, and hemodialysis was reintroduced when he was 44 years old. We gradually tapered the immu-

c Transmural endarteritis was recognized in the artery (Masson, $\times 200)$. d1 C4d staining was positive along peritubular capillaries $(\mathrm{C} 4 \mathrm{~d}, \times 400)$. d2 $\mathrm{C} 4 \mathrm{~d}$ staining was positive along endothelial cells of large arteries (C4d, $\times 400)$.

nosuppressants and stopped them after 1 year. Two months later, the patient noticed a slight fever and left lower abdominal pain, and microscopic hematuria developed. Abdominal computed tomography showed that the fat tissue in the hilum of the transplanted kidney was obscured, and the transplanted kidney was enlarged. Urinary tract infection was suspected initially, but the patient did not respond to antibiotics. He was then hospitalized with repeated obstruction of arteriovenous graft. On examination, he had a blood pressure of 139/94 $\mathrm{mm} \mathrm{Hg}$, a pulse rate of 77 beats $/ \mathrm{min}$, and a temperature of $37.0^{\circ} \mathrm{C}$. On physical exam, the transplanted kidney was found in the left lower abdomen, and tenderness was observed. Blood tests showed anemia (hemoglobin $8.2 \mathrm{~g} / \mathrm{dL}$ ) and increased fibrinogen $(510 \mathrm{mg} / \mathrm{dL})$. The C-reactive protein (CRP) was elevated to $5.27 \mathrm{mg} / \mathrm{dL}$. Other blood test results are as follows: WBC 7,400/ $\mu \mathrm{L}, \mathrm{Hb} 8.2 \mathrm{~g} / \mathrm{dL}$, Ht $26.0 \%$, Plt $16.0 \times 10^{4} / \mu \mathrm{L}, \mathrm{UN} 77$ $\mathrm{mg} / \mathrm{dL}, \mathrm{Cr} 17.09 \mathrm{mg} / \mathrm{dL}, \mathrm{Fbg} 510 \mathrm{mg} / \mathrm{dL}$, and D-dimer $1.6 \mu \mathrm{L} / \mathrm{mL}$. 

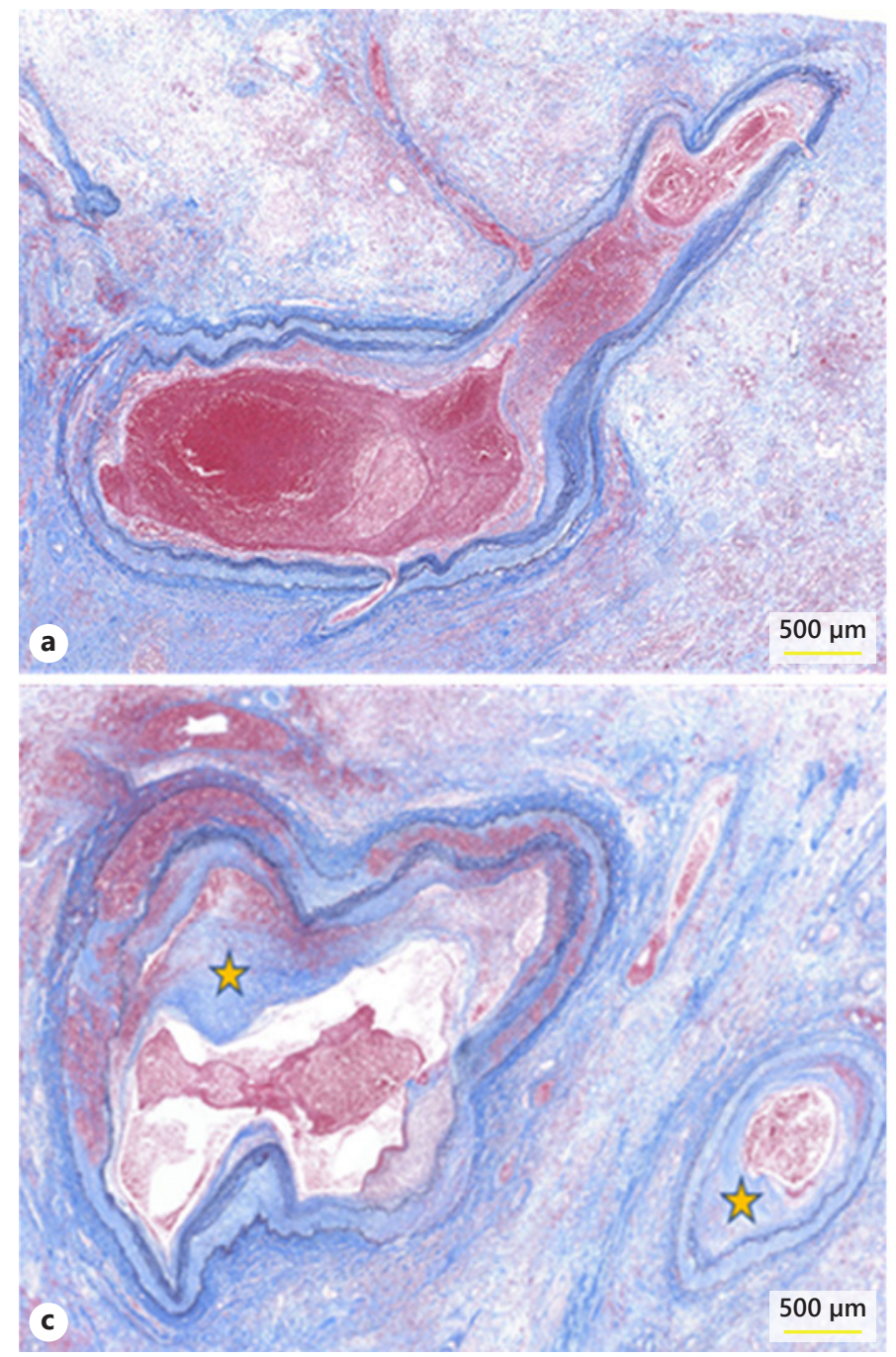

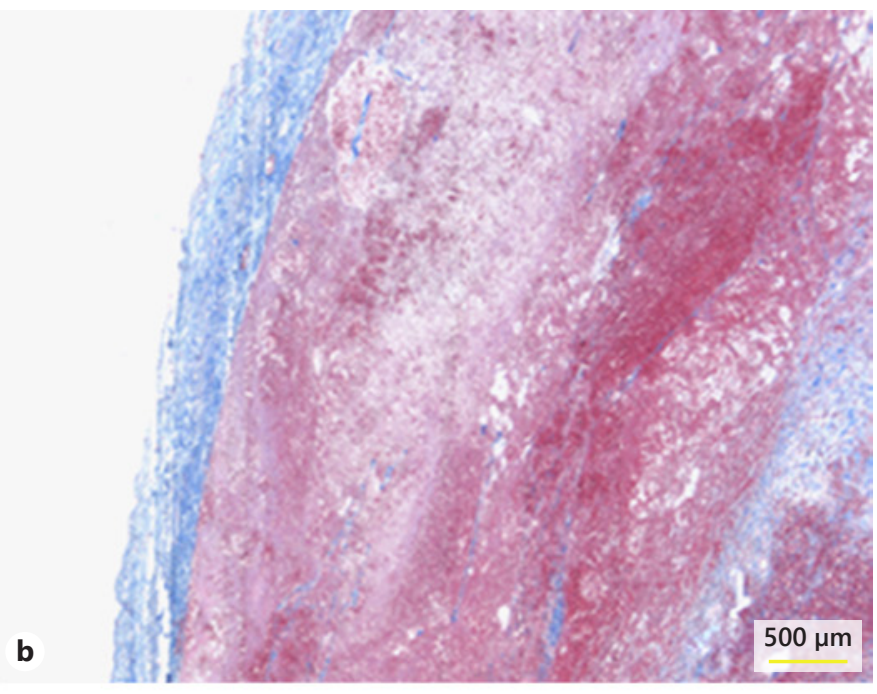

Fig. 3. a The segmental artery lumen was occluded by massive thrombi (Masson, $\times 40$ ). b Extensive hemorrhagic necrosis under the capsule extended in the same section as in (a) (Masson, $\times 40$ ). c At the level of segmental arteries, advanced chronic vasculopathy ( 3 ) and edematous inflammatory changes in the intima were observed (Masson, $\times 40$ ).
Blood and urine cultures were negative. Based on the clinical course, physical findings, and blood and imaging tests, the patient was diagnosed with graft intolerance syndrome. Oral mycophenolate mofetil was resumed at $250 \mathrm{mg}$ /day. However, his symptoms did not improve. Therefore, the immunosuppressive therapy was intensified, and steroid half-pulse therapy (methylprednisolone $500 \mathrm{mg} /$ day for 3 days) was added for 3 days. After the pulse therapy, the CRP decreased, but there was little symptom improvement, and the CRP increased again. The arteriovenous graft reoccluded and graft reconstruction was considered desirable, but the patient's abdominal pain and slight fever persisted, and prolonged high level of the CRP ( $\geq 4.0 \mathrm{mg} / \mathrm{dL})$ was also observed. Therefore, we decided to perform a nephrectomy to improve his condition. Transplant nephrectomy was performed in March 2018. Postoperatively, his symptoms disappeared without immunosuppressants, and the CRP decreased. The erythropoietin-resistant anemia also improved. An arteriovenous graft replacement was performed on the left forearm on June 19. Subsequently, no shunt occlusion was observed. The clinical course is shown in Figure 1.
The pathological findings are shown below. The extracted kidney weight had increased to $242 \mathrm{~g}$. The parenchyma was white with hemorrhagic areas suggesting necrosis, and the parenchyma was weakened in places (Fig. 2a). There was diffuse interstitial fibrosis and tubular atrophy in the cortex and diffuse infiltration of inflammatory cells, mainly mononuclear cells. In the medulla, severe interstitial hemorrhage was observed in places.

Most of the glomeruli showed global sclerosis, and severe transplant glomerulopathy was present in the remaining glomeruli (Fig. 2b). In the arteries, advanced arterial endarteritis was observed (Fig. 2c). The peritubular capillaries and endothelial cells of large arteries were C4d positive (Fig. 2d1, d2). Luminal obstruction due to massive thrombi was observed in the segmental arteries (Fig. 3a). In the same section, there was extensive hemorrhagic necrosis under the capsule (Fig. 3b). In larger vessels at the level of the segmental arteries, advanced chronic vasculopathy and edematous inflammatory changes in the intima were observed (Fig. 3c). 


\section{Discussion}

We report a case of graft intolerance syndrome in which transplant nephrectomy was performed 11 years after kidney transplantation. This case suggests the appropriate timing and necessity of transplant nephrectomy due to graft intolerance syndrome with persistent active allograft rejection.

In the case of early graft loss (often within 6 months), there are many vascular lesions, including arterial thrombosis and acute severe rejection [8]. These are related to kidney allograft rupture and rapid nephrectomy [9]. While transplant nephrectomy is very effective on persistent active allograft rejection, it is associated with greater mortality and morbidity, primarily due to bleeding and infection [11]. The likelihood of producing antibodies against human leukocyte antigens is also increased. Therefore, transplant nephrectomy is rarely performed in patients who lose kidney function $>6$ months after kidney transplantation. Thus, the decision to perform a transplant nephrectomy should be made carefully after considering the risks and benefits. Watchful waiting is usually selected when dialysis therapy has been resumed, and there is no urgent reason to perform a transplant nephrectomy. Our case involved late graft loss. The indications for transplant nephrectomy after late graft failure are as follows: space for reimplantation, complete immediate discontinuation of immunosuppressants, malignant tumor in the transplanted kidney, graft intolerance syndrome, and risk of renal rupture $[12,13]$.

Graft intolerance syndrome, defined as a condition in which the patient is unable to tolerate the allografted kidney(s) after loss of function, is the main indication for transplant nephrectomy after late kidney graft failure. The symptoms include fever, flulike symptoms, malaise, hematuria, localized pain, renal transplant enlargement, and refractory anemia with elevated CRP. The diagnosis is made after ruling out infection. Most cases occur within 1 year after the cessation of renal function, with an incidence of 30-50\% [14]. Research has examined the risk of transplant nephrectomy due to graft intolerance syndrome after kidney transplantation in which the kidney function failed after $>6$ months. That study reported that older donor age, the number of rejections, and shorter kidney transplant graft survival were risk factors for transplant nephrectomy [15]. In our case, although the transplanted kidney survived for 10 years, the donor was 68 years old. Furthermore, prolonged inflammation due to active chronic antibody-mediated rejection was considered the reason of nephrectomy. Pathologically, the re- sected kidney graft showed severe transplant glomerulopathy, endarteritis, and C4d-positivity in the peritubular capillaries that indicated persistent active antibody-mediated rejection 2 years after kidney graft loss. In addition, the massive thrombi in the larger arteries, including some segmental and interlobar arteries, might be related to severe endothelial cell damage, resulting from the persistent allograft rejection. These thrombi induced widespread cortical necrosis. The gross pathology showed that the kidney graft parenchyma was vulnerable in some places, which might cause future kidney graft rupture. The patient's condition was in a state of lull, but considering the pathological findings, it was considered that his life would have been in danger due to renal rupture if he had been left as it was. Furthermore, by performing nephrectomy, the arteriovenous graft can be reconstructed and stable dialysis can be performed. In consideration of the above, the timing of this extraction was considered appropriate.

In conclusion, preemptive graft nephrectomy is not common in patients $>6$ months after transplantation. However, it remains an effective option in some cases of severe graft intolerance syndrome. This is true even when the posttransplantation interval is relatively long, as in the case presented above.

\section{Statement of Ethics}

This case report has been approved by the research ethics committee of The Jikei University School of Medicine.

\section{Conflict of Interest Statement}

The authors have no conflict of interest to declare.

The English in this document has been checked by at least 2 professional editors, both native speakers of English. For a certificate, please see: http://www.textcheck.com/certificate/KQz5de, http://www.textcheck.com/certificate/PXnHmT.

\footnotetext{
References

1 Morales A, Gavela E, Kanter J, Beltrán S, Sancho A, Escudero V, et al. Treatment of renal transplant failure. Transplant Proc. 2008; 40(9):2909-11.

2 Lamb KE, Lodhi S, Meier-Kriesche HU. Long-term renal allograft survival in the United States: a critical reappraisal. Am J Transplant. 2011;11(3):450-62.

3 Gregoor PJ, Kramer P, Weimar W, van Saase JL. Infections after renal allograft failure in patients with or without low-dose maintenance immunosuppression. Transplantation. 1997;63(10):1528-30.
}

Tomonari et al. 
4 López-Gómez JM, Pérez-Flores I, Jofré R, Carretero D, Rodríguez-Benitez P, Villaverde $\mathrm{M}$, et al. Presence of a failed kidney transplant in patients who are on hemodialysis is associated with chronic inflammatory state and erythropoietin resistance. J Am Soc Nephrol. 2004;15(9):2494-501.

5 Ojo A, Wolfe RA, Agodoa LY, Held PJ, Port FK, Leavey SF, et al. Prognosis after primary renal transplant failure and the beneficial effects of repeat transplantation: multivariate analyses from the United States Renal Data System. Transplantation. 1998;66(12):1651-9.

6 Ayus JC, Achinger SG, Lee S, Sayegh MH, Go AS. Transplant nephrectomy improves survival following a failed renal allograft. J Am Soc Nephrol. 2010;21(2):374.

7 Shapiro DJ, Blumenkrantz MJ, Shinaberger $\mathrm{JH}$, Coburn JW. Useful function of "nonfunctioning” renal homograft. Br Med J. 1975; 3(5976): 140 .
8 Vanrenterghem $\mathrm{Y}$, Khamis S. The management of the failed renal allograft. Nephrol Dial Transplant. 1996 Jun;11(6):955-7.

9 Abouljoud MS, Deierhoi MH, Hudson SL, Diethelm AG. Risk factors affecting second renal transplant outcome, with special reference to primary allograft nephrectomy. Transplantation. 1995;60(2):138-44.

10 Akoh JA. Transplant nephrectomy. World J Transplant. 2011;1(1):4-12.

11 Vanrenterghem Y, Khamis S. The management of the failed renal allograft. Nephrol Dial Transplant. 1996;11(6):955-7.

12 Secin FP, Rovegno AR, del Rosario Brunet M, Marrugat RE, Dávalos Michel M, Fernandez $\mathrm{H}$. Cumulative incidence, indications, morbidity and mortality of transplant nephrectomy and the most appropriate time for graft removal: only nonfunctioning transplants that cause intractable complications should be excised. J Urol. 2003;169(4):1242-6.
13 Andrews PA; Standards Committee of the British Transplantation Society. Summary of the British transplantation Society Guidelines for management of the failing kidney transplant. Transplantation. 2014;98(11):1130-3.

14 Delgado P, Diaz F, Gonzalez A, Sanchez E, Gutierrez P, Hernandez D, et al. Intolerance syndrome in failed renal allografts: incidence and efficacy of percutaneous embolization. Am J Kidney Dis. 2005;46(2):339-44.

15 Kim L, Verhoeks CM, van den Brand JAJG, Hilbrands LB. Graft intolerance syndrome requiring graft nephrectomy after late kidney graft failure: can it be predicted? A retrospective cohort study. Transplant Int. 2018;31: 220-9. 